\title{
Stem cells and tissue niche: two faces of the same coin of muscle regeneration
}

\author{
Bianca Maria Scicchitano (1), Gigliola Sica (1), Antonio Musarò (2,3)
}

(1) Institute of Histology and Embryology, Catholic University School of Medicine, Rome, Italy; (2) Institute Pasteur Cenci-Bolognetti; DAHFMO-Unit of Histology and Medical Embryology, IIM; Sapienza University of Rome, Italy ;(3) Center for Life Nano Science@Sapienza, Istituto Italiano di Tecnologia, Italy

This article is distributed under the terms of the Creative Commons Attribution Noncommercial License (CC BY-NC 4.0) which permits any noncommercial use, distribution, and reproduction in any medium, provided the original author(s) and source are credited.

\begin{abstract}
Capacity of adult muscle to regenerate in response to injury stimuli represents an important homeostatic process. Regeneration is a highly coordinated program that partially recapitulates the embryonic developmental program. However, muscle regeneration is severely compromised in several pathological conditions. It is likely that the restricted tissue repair program under pathological conditions is due to either progressive loss of stem cell populations or to missing signals that limit the damaged tissues to efficiently activate a regenerative program. It is therefore plausible that loss of control over these cell fates might lead to a pathological cell transdifferentiation, limiting the ability of a pathological muscle to sustain an efficient regenerative process. The critical role of microenvironment on stem cells activity and muscle regeneration is discussed.
\end{abstract}

Key Words: muscle regeneration, tissue niche, muscle pathologies, satellite cells, IGF-1

Muscle regeneration is a coordinated process in which several factors are sequentially activated to maintain and preserve muscle structure and function upon injured stimuli. Although adult skeletal muscle is composed of fully differentiated fibers, it retains the capacity to regenerate in response to injury and to modify its contractile and metabolic properties in response to changing demand. ${ }^{1}$ Regeneration is an important homeostatic process, which guarantees the maintenance of muscle integrity and plasticity. Muscle regeneration and repair occur in four interrelated and time-dependent phases: degeneration, inflammation, regeneration, and remodelling/maturation (Figure 1A). ${ }^{1,2}$ Injury of myofibers results in the rapid necrosis, which activates a defined inflammatory response (Figure 1), characterized by the recruitment of specific myeloid cell populations within the injured area. ${ }^{3}$ Specifically, neutrophils represent the first inflammatory myeloid cells that invade the site of muscle injury; the number of neutrophils usually drops 24 hours after damage and they are normally no longer detectable after 36-48 hours post injury (Figures 1). ${ }^{4,5}$ The production of soluble interleukin-6 receptor (sIL-6R) by neutrophils regulates the change from a neutrophilic to macrophages infiltration. Macrophages rapidly increase within 24 hours after injury (Figure 1) and they are the predominant inflammatory cell type within the injured area. M1 and M2 nomenclature is usually used to refer to the two extremes of a spectrum of possible forms of macrophage activation. ${ }^{6,7}$ In particular, it has been proposed that macrophages develop into either type 1 inflammatory (M1) or type 2 anti-inflammatory (M2) subsets and that macrophages sequentially change their functional phenotype in response to changes in microenvironmental influences. ${ }^{6-8}$. M1 macrophages remove tissue debris, whereas M2 macrophages modulate the immune responses and activate stem cell populations. ${ }^{7,8}$ Thus, the inflammatory response is a coordinate process that must be finely regulated to obtain an efficient regenerative process. The inflammatory response is followed by regenerative phase (Figure 1A), characterized by satellite cells activation and by the presence of regenerating fibers, which can be morphologically distinguishable by the presence of characteristic central nuclei and by the expression of the embryonic/neonatal isoform of myosin heavy chain (MyHC). ${ }^{9,10}$ The final phase is a period during which the maturation of the regenerated myofibers, the contraction and reorganization of the scar tissue (remodelling of extracellular matrix) (Figure 1A), and the recovery of the functional performance of injured muscle occur. ${ }^{11}$ 


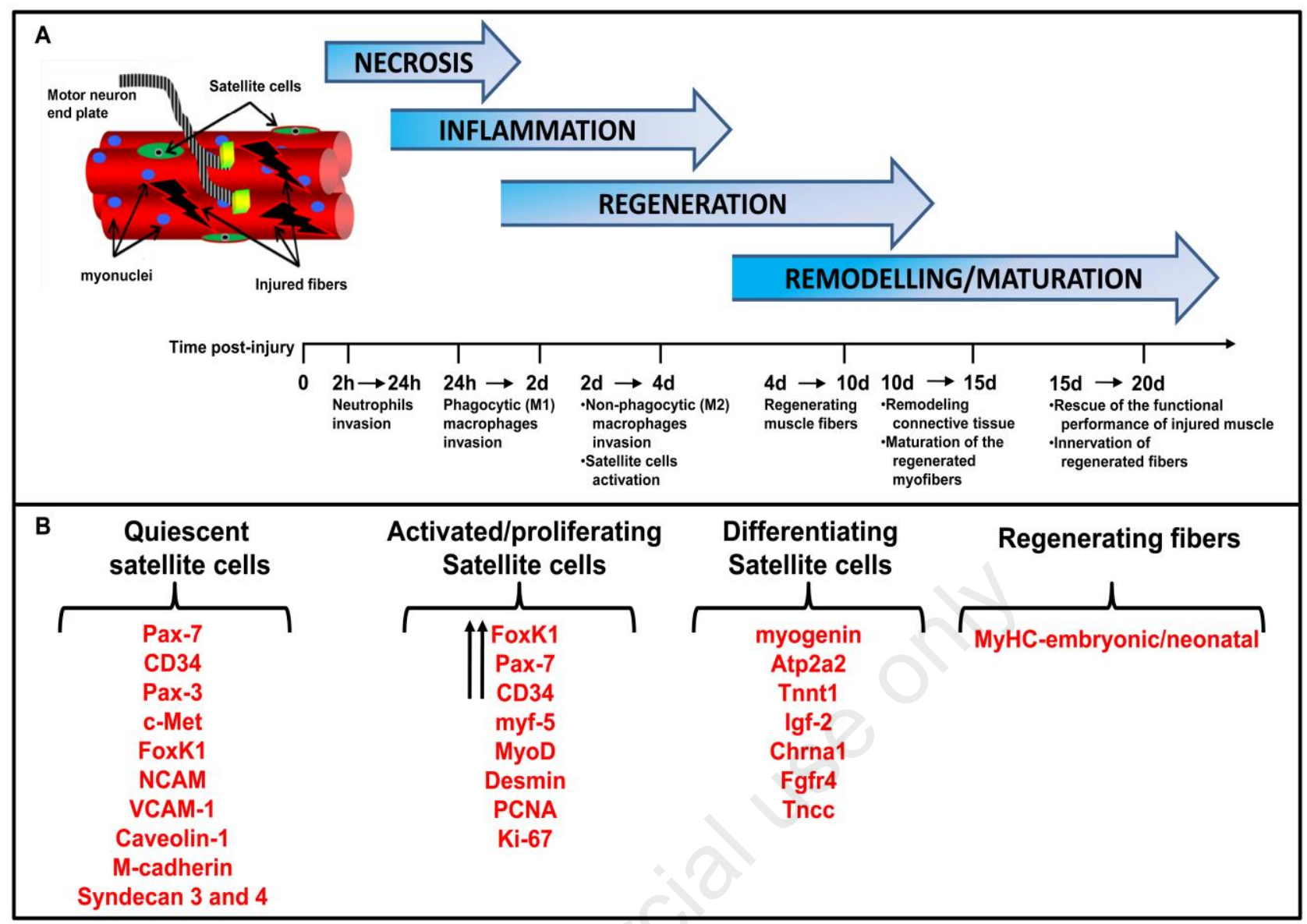

Fig. 1 Model of stem cell-mediated muscle regeneration. (A) Schematic representation of the four interrelated and time-dependent phases underlying muscle regeneration. The relevant biological responses, activated after cardiotoxin (CTX) injection, are indicated. (B) Schematic model outlining the relevant markers expressed by satellite cells during the different stages of regeneration. (h: hours; d: days).

The role of satellite cells and non-muscle stem cells on muscle regeneration

The dominant role in muscle homeostasis and regeneration is played by satellite cells, ${ }^{12}$ which reside between the basal lamina and sarcolemma of myofibers. Satellite cells can be activated in response to both physiological stimuli, such as exercise, and under pathological conditions, such as injury and degenerative diseases, to generate a committed population of myoblasts that are capable of fusion and differentiation. ${ }^{13}$ Satellite cells are able to fuse with existing myofibers, repairing damaged muscle fibers, or alternatively fuse to each other to form new myofibers ${ }^{1}$. RT-PCR analysis, gene targeting strategies, and molecular imaging revealed that satellite cells present a heterogeneous profile of gene expression depending on the functional stage of the myogenic program. It has been reported that quiescent satellite cells express several relevant markers such as c-Met, M-cadherin, FoxK, Pax-7, NCAM, syndecan 3 and 4, CD34, caveolin-1, Sox 8, Sox 15, and VCAM-1 (Figure 1B). ${ }^{1}$ Pax3, the paralog (pair of genes that derive from the same ancestral gene) of $\operatorname{Pax} 7$, is also expressed in quiescent muscle satellite cells in a subset of muscles and it plays an important role in regulating the entry of satellite cells into the myogenic programme. ${ }^{14,15}$ Once activated, satellite cells up-regulate c-Met, Pax-7, and $\mathrm{M}$-cadherin and activate the expression factors involved in the specification of the myogenic program such as myf-5 and MyoD (Figure 1B). The activated satellite cells proliferate as indicated by the expression of factors involved in cell cycle progression (Figure 1B) ${ }^{1}$ and by incorporation of $\mathrm{BrDU}$ and $[3 \mathrm{H}]$ thimidine. Ultimately the committed satellite cells fuse each other, or to the existing fibers, to form new muscle fibers, which are characterized by small size and by the expression of the embryonic/neonatal isoform of MyHC (Figure 1B). More recently, it has been suggested that other "nonmuscle" stem cell populations can participate to muscle regeneration, contributing to maintain the pool of satellite cells. These stem cell populations could either reside within muscle, or recruited via circulation in response to homing signals secreted by injured skeletal muscle. These populations include endothelialassociated cells, ${ }^{1-6}$ interstitial cells, ${ }^{17-19}$ and bone marrow-derived cells. ${ }^{20,21}$ At first sight the origin of non 
muscle-derived stem cells appears to be mainly restricted to the hemo-vascular system (hematopoietic, endothelial, pericytes). A question relevant to muscle regeneration is whether there is any lineage relationship between one or more types of mesoderm stem cells and muscle satellite cells. Although there is not a conclusive answer to this question, it is possible that any of these cells may leave the vessel wall, enter the interstitial space and produce factors that stimulate the activity of satellite cells.

\section{The role of tissue niche on muscle regeneration}

One of the critical points that remain to be addressed is the following: if skeletal muscle possesses a stem cell compartment, it is not clear why skeletal muscle fails to regenerate under pathological conditions. Either the resident muscle stem cells drastically decrease during aging and in several degenerative diseases or perhaps the pathological muscle is a prohibitive environment for stem cells activation and function. Although we lack definitive answers, several evidences suggested that with age or under pathological conditions, the systemic environment impinges the activity of satellite cells and stimulates fibrotic accumulation. In particular, recent reports described the identification of muscle-derived interstitial cells, referred to as fibro-adipocyte progenitors (FAPs). ${ }^{22}$ Under physiologic regenerative stimuli, FAPs secrete factors that promote satellite cellmediated regeneration. ${ }^{23}$ In contrast, in degenerating muscles, such as muscular dystrophy, these cells turn into fibro-adipocytes, which mediate fat deposition and fibrosis. $^{24}$

The environmental niche that nurtures and maintains the stem cells at different anatomical sites and influences stem cell proliferation and differentiation represents a critical component of muscle regeneration. Indeed, heterochronic experiments demonstrated that old muscle successfully regenerates when transplanted in a young animal, ${ }^{25}$ whereas the regeneration of young muscle transplanted in an old host was impaired. This hypothesis has been clearly validated by parabiotic experiments, the union of two organisms that share the circulatory system, demonstrating the rejuvenation of aged progenitor cells by exposure to a young systemic environment. ${ }^{26}$

Similarly, we also demonstrated that human satellite cells fail to differentiate when cultured in isochronic conditions. ${ }^{27}$ Immunofluorescence analysis for the expression of MyHC revealed that aged satellite cells did not display major defect in the propensity to fuse when differentiated under standard conditions, namely in DMEM supplemented with 5\% horse serum. Of note, we observed that autologous serum (isochronic culture conditions) dramatically reduced muscle differentiation, which was partially rescued when aged satellite cells were differentiated in heterologous/ heterochronic serum (from young donors).$^{27}$ These data suggest that with age, the systemic environment is less effective in maintaining the myogenic fate of muscle stem cells and, instead, facilitates conversion to a fibrogenic fate. The modifications with aging in the secretome of the different cell types present at different phases of regeneration is probably then crucial, as is expression of relevant cell surface receptors, which can also change with age. Among potential factors, Notch, Wnt, and Insulin-like growth factor-1 (IGF-1) may modulate the pathological niche. Notch signaling is one of the major pathway that regulates the activation and expansion of the satellite cell lineage during embryogenesis ${ }^{28}$ and in the adult. ${ }^{29}$ The decline of Notch signaling with age is thought to be one of the causes of the decreased regenerative potential of aged satellite cells. ${ }^{26}$ Indeed forced activation of Notch signaling in injured muscle of aged mice restores regenerative potential to that tissue. $^{30}$ In addition, the temporal balance between Notch and Wnt signaling orchestrates the precise progression of muscle precursor cells along the myogenic lineage pathway, during postnatal myogenesis. ${ }^{31}$ Nevertheless, the Wnt signal must be finely modulated, since a persistence of Wnt signal, as, for example, observed in aged mice, can be associated with an increase in tissue fibrosis. ${ }^{32-34}$ Conversely, the myogenic-to-fibrogenic conversion has been abrogated by Wnt inhibitors.

Among growth factors, insulin-like growth factor-1 (IGF-1) has been implicated in many anabolic pathways in skeletal muscle and it plays a central role during muscle regeneration. ${ }^{35-37}$ Unlike other growth factors, the local isoform of IGF-1 (mIGF-1) also stimulates myogenic differentiation, generates a pronounced muscle hypertrophy, ${ }^{38-41}$ modulates the inflammatory response, and accelerates the functional rescue of injured skeletal muscle. ${ }^{36,42,43}$

In addition, we observed that forced expression of mIGF-1 improves muscle mass and strength, decreases myonecrosis, and reduces fibrosis in dystrophic mice. ${ }^{44}$ This is of particular relevance to the human dystrophic condition where virtually all skeletal muscles succumb to fibrosis. In addition we recently demonstrated that muscle-specific expression of mIGF-1 can counter aspects of the muscular dystrophy associated with the loss of dystrophin, modulating relevant molecules of the genetic and epigenetic circuitries in the mdx dystrophic mouse model. ${ }^{45}$ Our work is consistent with a model in which overexpression of mIGF-1 confers robustness to dystrophic muscle, impedes the activation of a chronic inflammatory response, activates the circuitry of muscle differentiation and maturation. This results in a functional homeostatic maintenance of dystrophic muscle. ${ }^{45}$ Another study also demonstrated that coinjection of the rAAV-microdystrophin and rAAVmIGF-1 vectors resulted in increased muscle mass and strength, reduced myofiber degeneration, and increased protection against contraction-induced injury. ${ }^{46}$ These results suggest that a combination of promoting muscle regenerative capacity and preventing muscle necrosis 
could be an effective treatment for the secondary symptoms caused by the primary loss of dystrophin.

In addition, localized expression of mIGF-1 delays the onset and progression of disease in amyotrophic lateral sclerosis (ALS) mice, ${ }^{47-50}$ ameliorates muscle pathology and reduces motor neuron loss in a mouse model of spinal and bulbar muscular atrophy (SBMA),$^{51}$ and improves muscle mass and extends the survival of severe spinal muscular atrophy (SMA) mice. ${ }^{52}$

Interestingly, we recently demonstrated that IGF-1 isoforms are also up-regulated in senior patients treated with electrical stimulation (ES), ${ }^{53}$ promoting satellite cells activation, reduction in the expression of musclespecific atrophy-related ubiquitin ligase genes, and promoting the remodeling of extracellular matrix. ${ }^{54}$

Another potential factor that, similarly to IGF- $1,{ }^{55}$ plays important physiological roles in skeletal muscle development and homeostasis is the neurohypophyseal hormone vasopressin (AVP). In a recent study, ${ }^{56}$ we demonstrated that muscle-specific stimulation of AVP induces acceleration of satellite cell activation and earlier stimulation of specific pathways involved in muscle regeneration.

\section{Conclusions}

These findings demonstrate that while stem cells represent an important determinant for tissue regeneration, a "qualified" environment is necessary to guarantee and achieve functional results.

Thus, in several muscle pathologies the systemic environment is less effective in maintaining the myogenic fate of muscle stem cells and, instead, facilitates conversion to a fibrogenic fate. In this context, therapeutic applications of adult stem cells to aged or pathological tissue repair, in the context of regenerative medicine, ${ }^{57-63}$ will require an increased understanding of stem-cell biology, environment of pathological tissue and interaction between the two.

\section{Author's contributions}

BMS, GS, and AM organized the work and wrote the paper.

\section{Acknowledgments}

Work in the authors' laboratories has been supported by Telethon (GGP14066) and Fondazione Roma.

\section{Conflict of Interest}

The authors declare no potential conflict of interests.

\section{Corresponding Author}

Antonio Musarò, Unit of Histology and Medical Embryology, Via A. Scarpa 14, I-00161, Rome Italy e-mail: antonio.musaro@uniroma1.it

\section{E-mails of coAuthors}

Bianca Maria Scicchitano: biancamaria.scicchitano@unicatt.it

Gigliola Sica: gigliola.sica@ unicatt.it

\section{References}

1. Musarò A. The Basis of Muscle Regeneration. Advances in Biology 2014, 2014:1-16.

2. Carosio S, Berardinelli MG, Aucello M, Musarò A. Impact of ageing on muscle cell regeneration. Ageing Res. Rev 2011;10:35-42.

3. Tidball JG. Inflammatory processes in muscle injury and repair. Am J Physiol Regul Integr Comp Physiol 2005;288:R345-53.

4. Fielding RA, Manfredi TJ, Ding W, et al. Acute phase response in exercise. III. Neutrophil and IL1 beta accumulation in skeletal muscle. Am J Physiol 1993;265(1 Pt 2):R166-72.

5. Belcastro AN, Arthur GD, Albisser TA, Raj DA. Heart, liver, and skeletal muscle myeloperoxidase activity during exercise. J Appl Physiol 1996;80:1331-35.

6. Geissmann F, Jung S, Littman DR. Blood monocytes consist of two principal subsets with distinct migratory properties. Immunity 2003;19:71-82.

7. Mantovani A, Sica A, Sozzani S, et al. The chemokine system in diverse forms of macrophage activation and polarization. Trends Immunol 2004;25:677-86.

8. Deng B, Wehling-Henricks M, Villalta SA, et al. IL-10 triggers changes in macrophage phenotype that promote muscle growth and regeneration. J Immunol 2012;189:3669-80.

9. Karpati G, Carpenter S, Prescott S. Small-caliber skeletal muscle fibers do not suffer necrosis in mdx mouse dystrophy. Muscle Nerve 1988;11:795-803.

10. Karpati G, Molnar MJ. Muscle fibre regeneration in human skeletal muscle diseases. In: Schiaffino, S., Partridge, T., (Eds.). Skeletal Muscle Repair and Regeneration. (Advances in Muscle Research) Springer Verlag, The Netherlands, 2008; pp. 45-64.

11. Goetsch SC, Hawke TJ, Gallardo TD, et al. Transcriptional profiling and regulation of the extracellular matrix during muscle regeneration. Physiol Genomics 2003;14: 261-71.

12. Mauro A. Satellite cell of skeletal muscle fibers. J Biophys Biochem Cytol 1961;9:493-5.

13. Gayraud-Morel B, Chrétien F, Tajbakhsh S. Skeletal muscle as a paradigm for regenerative biology and medicine. Regen Med 2009;4:293319.

14. Relaix F, Montarras D, Zaffran S, et al. Pax3 and Pax7 have distinct and overlapping functions in adult muscle progenitor cells. J Cell Biol 2006;172:91-102.

15. Buckingham M. Skeletal muscle progenitor cells and the role of Pax genes. C R Biol 2007;330:530 3.

16. De Angelis L, Berghella L, Coletta M, et al. Skeletal myogenic progenitors originating from 


\section{Stem cells and tissue niche in muscle regeneration}

Eur J Transl Myol 26 (4): 333-338

embryonic dorsal aorta coexpress endothelial and myogenic markers and contribute to postnatal muscle growth and regeneration. J Cell Biol 1999;147:869-78.

17. Kuang S, Chargé SB, Seale P, et al. Distinct roles for $\operatorname{Pax} 7$ and $\operatorname{Pax} 3$ in adult regenerative myogenesis. J Cell Biol 2006;172:103-13.

18. Polesskaya A, Seale P, Rudnicki MA. Wnt signaling induces the myogenic specification of resident $\mathrm{CD} 45+$ adult stem cells during muscle regeneration. Cell 2003;113:841-52.

19. Tamaki T, Akatsuka A, Ando K, et al. Identification of myogenic-endothelial progenitor cells in the interstitial spaces of skeletal muscle. J Cell Biol 2002;157:571-7.

20. Asakura A, Seale P, Girgis-Gabardo A, Rudnicki MA. Myogenic specification of side population cells in skeletal muscle. J Cell Biol 2002;159:12334.

21. Gussoni E, Soneoka Y, Strickland CD, et al. Dystrophin expression in the mdx mouse restored by stem cell transplantation. Nature 1999;401:390-4.

22. Uezumi A, Ito T, Morikawa D, et al. Fibrosis and adipogenesis originate from a common mesenchymal progenitor in skeletal muscle. J Cell Sci 2011;124:3654-64.

23. Joe AW, Yi L, Natarajan A, et al. Muscle injury activates resident fibro/adipogenic progenitors that facilitate myogenesis. Nat Cell Biol 2010;12:15363.

24. Uezumi A, Fukada S, Yamamoto $\mathrm{N}$, et al. Mesenchymal progenitors distinct from satellite cells contribute to ectopic fat cell formation in skeletal muscle. Nat Cell Biol 2010;12:143-52.

25. Carlson BM, Faulkner JA. Muscle transplantation between young and old rats: age of host determines recovery. Am J Physiol 1989;256:C1262-6.

26. Conboy IM, Conboy MJ, Wagers AJ, et al. Rejuvenation of aged progenitor cells by exposure to a young systemic environment. Nature 2005;433:760-4.

27. Barberi L, Scicchitano BM, De Rossi M, et al. Age-dependent alteration in muscle regeneration: the critical role of tissue niche. Biogerontology 2013;14:273-92.

28. Vasyutina E, Lenhard DC, Birchmeie C. Notch function in myogenesis. Cell Cycle 2007;6:1451-4.

29. Conboy IM, Rando TA. The regulation of Notch signaling controls satellite cell activation and cell fate determination in postnatal myogenesis. Dev Cell 2002;3:397-409.

30. Conboy IM, Conboy MJ, Smythe GM, Rando TA. Notch-mediated restoration of regenerative potential to aged muscle. Science 2003;302:15757.
31. Brack AS, Conboy IM, Conboy MJ, et al. A temporal switch from notch to Wnt signaling in muscle stem cells is necessary for normal adult myogenesis. Cell Stem Cell 2008;2:50-9.

32. Chilosi M, Poletti V, Zamò A, et al. Aberrant Wnt/beta-catenin pathway activation in idiopathic pulmonary fibrosis. Am J Pathol 2003;162:14951502.

33. Brack AS, Conboy MJ, Roy S, et al. Increased Wnt signaling during aging alters muscle stem cell fate and increases fibrosis. Science 2007;317:80710 .

34. Jiang F, Parsons CJ, Stefanovic B. Gene expression profile of quiescent and activated rat hepatic stellate cells implicates Wnt signaling pathway in activation. J Hepatol 2006;45:401-9.

35. Musarò $A$, Rosenthal $\mathrm{N}$. The critical role of Insulin-like Growth Factor-1 isoforms in the physiopathology of skeletal muscle. Curr Genomics 2006;7:19-32.

36. Scicchitano BM, Rizzuto E, Musarò A. Counteracting muscle wasting in aging and neuromuscular diseases: the critical role of IGF-1. Aging 2009;1:451-7.

37. Shavlakadze T, Winn N, Rosenthal N, Grounds MD. Reconciling data from transgenic mice that overexpress IGF-I specifically in skeletal muscle. Growth Horm IGF Res 2005;15:4-18.

38. Musarò A, Rosenthal N. Maturation of the myogenic program is induced by postmitotic expression of insulin-like growth factor I. Mol Cell Biol 1999;19:3115-24.

39. Musarò A, McCullagh KJ, Naya FJ, et al. IGF-1 induces skeletal myocyte hypertrophy through calcineurin in association with GATA-2 and NFATc1. Nature 1999;400:581-5.

40. Musarò A, McCullagh K, Paul A, et al. Localized Igf-1 transgene expression sustains hypertrophy and regeneration in senescent skeletal muscle. Nat Genet 2001;27:195-200.

41. Barton-Davis ER, Shoturma DI, Musarò A, et al. Viral mediated expression of insulin-like growth factor I blocks the aging-related loss of skeletal muscle function. Proc Natl Acad Sci U S A 1998;95:15603-7.

42. Musarò A, Giacinti C, Borsellino G, et al. Stem cell-mediated muscle regeneration is enhanced by local isoform of insulin-like growth factor 1 . Proc Natl Acad Sci U S A 2004;101:1206-10.

43. Pelosi L, Giacinti C, Nardis C, et al. Local expression of IGF-1 accelerates muscle regeneration by rapidly modulating inflammatory cytokines and chemokines. FASEB J 2007;21:1393-1402.

44. Barton ER, Morris L, Musaro A, et al. Muscle specific expression of Insulin-like Growth Factor I counters muscle decline in mdx mice. J Cell Biol 2002;157:137-47. 
45. Pelosi L, Coggi A, Forcina L, Musarò A. MicroRNAs modulated by local mIGF-1 expression in mdx dystrophic mice. Front Aging Neurosci. 2015;769.

46. Abmayr S, Gregorevic P, Allen JM, Chamberlain JS. Phenotypic improvement of dystrophic muscles by $\mathrm{rAAV} /$ microdystrophin vectors is augmented by Igf1 codelivery. Mol Ther 2005;12:441-50.

47. Dobrowolny G, Giacinti C, Pelosi L, et al. Muscle expression of a local Igf-1 isoform protects motor neurons in an ALS mouse model. J Cell Biol 2005;168:193-9.

48. Dobrowolny G, Aucello M, Molinaro M, Musarò A. Local expression of mIgf-1 modulates ubiquitin, caspase and CDK5 expression in skeletal muscle of an ALS mouse model. Neurol Res 2008;30:131-6.

49. Kaspar BK, Lladó J, Sherkat N, Rothstein JD, Gage FH. Retrograde viral delivery of IGF-1 prolongs survival in a mouse ALS model. Science 2003;301:839-42.

50. Musarò A. Understanding ALS: new therapeutic approaches. FEBS J 2013;280:4315-22.

51. Palazzolo I, Stack C, Kong L, et al. Overexpression of IGF-1 in muscle attenuates disease in a mouse model of spinal and bulbar muscular atrophy. Neuron 2009;63:316-28.

52. Bosch-Marcé M, Wee CD, Martinez TL, et al. Increased IGF-1 in muscle modulates the phenotype of severe SMA mice. Hum Mol Genet 2011;20:1844-53.

53. Kern H, Barberi L, Löfler S, et al. Electrical stimulation counteracts muscle decline in seniors. Front Aging Neurosci. 2014;6:189. doi: 10.3389/fnagi.2014.00189.

54. Sandra Zampieri, Simone Mosole, Stefan Löfler, et al. Physical Exercise in Aging: Nine Weeks of Leg Press or Electrical Stimulation Training in 70 Years Old Sedentary Elderly People. Eur J Transl Myol 2015; 25: 237-242. doi: 10.4081/ejtm.2015.5374
55. Musarò A, Giacinti C, Pelosi L, et al. Stem Cellmediated muscle regeneration and repair in aging and neuromuscular diseases. Eur $\mathbf{J}$ Histochem 2007;51:35-43.

56. Toschi A, Severi A, Coletti D, et al. Skeletal muscle regeneration in mice is stimulated by local overexpression of V1a-vasopressin receptor. Mol Endocrinol 2011;25:1661-73.

57. Huang H, Sun T, Chen L, et al. Consensus of clinical neurorestorative progress in patients with complete chronic spinal cord injury. Cell Transplant 2014;23 Suppl 1:S5-17. doi: 10.3727/096368914X684952.

58. Kern H, Boncompagni S, Rossini K, et al. Longterm denervation in humans causes degeneration of both contractile and excitation- contraction coupling apparatus, wich is reversibile by functional electrical stimulation (FES). A role for myofiber regeneration? J Neuropathol Exp Neurol 2004;63:919-31.

59. Carraro U, Boncompagni S, Gobbo V, et al. Persistent muscle fiber regeneration in long term denervation. Past, present, future. Eur J Transl Myol. 2015;25:77-92. Doi: 10.4081/bam.2015.2.77

60. Carraro U, Kern H, Gava P, et al. Biology of Muscle Atrophy and of its Recovery by FES in Aging and Mobility Impairments: Roots and ByProducts. Eur J Transl Myol. 2015;25:221-30. doi: 10.4081/ejtm.2015.5272

61. Barberi L, Scicchitano BM, Musaro A. Molecular and Cellular Mechanisms of Muscle Aging and Sarcopenia and Effects of Electrical Stimulation in Seniors. Eur J Transl Myol 2015;25:231-6. doi: 10.4081/ejtm.2015.5227.

62. Forbes SJ, Rosenthal N. Preparing the ground for tissue regeneration: from mechanism to therapy. Nat Med 2014; 20:857-69. doi: 10.1038/nm.3653.

63. Bianco P, Barker R, Brüstle O, et al. Regulation of stem cell therapies under attack in Europe: for whom the bell tolls. EMBO J 2013;32:1489-95. doi: 10.1038/emboj.2013.114. 